Bull. Austral. Math. Soc.

Vol. 53 (1996) [447-454]

\title{
ALMOST FIXED POINT AND BEST APPROXIMATIONS THEOREMS IN $H$-SPACES
}

\author{
O. HADŽIĆ
}

Using the methods of KKM theory, almost fixed point and best approximations theorems in $H$-spaces are proved.

\section{INTRODUCTION}

The notion of an $H$-space was introduced by Bardaro and Ceppitelli in [1] and since 1988, many results from Nonlinear Functional Analysis in such spaces have been obtained $[1,2,3,4,5,6,7,13,14,15,16,17,18]$.

In this paper we shall introduce some generalisations of the Zima condition [8, 9, 10]) on a subset of an $H$-space. Some fixed point and almost fixed point theorems for single-valued and multi-valued mappings $f: K \rightarrow 2^{X}$, where $X$ is a not necessarily locally convex topological vector space and $K$ satisfies the Zima condition, have been proved in $[8,9,10]$.

\section{Preliminaries}

Let $A$ be a subset of a topological space $X$. By $2^{A}$ we denote the family of all nonempty subsets of $A$ and by $\mathcal{F}(A)$ the family of all nonempty finite subsets of $A$.

In [1] the following two definitions are given.

DEFINITION 1. A pair $\left(X,\left\{\Gamma_{A}\right\}\right)$ is said to be an $H$-space if $X$ is a topological space and $\left\{\Gamma_{A}\right\}_{A \in \mathcal{F}(X)}$ is a given family of contractible subsets $\Gamma_{A}$ of $X$, such that $A \subset B \subset X$ implies $\Gamma_{A} \subset \Gamma_{B}$.

DEFINITION 2. A nonempty subset $D$ of an $H$-space $\left(X,\left\{\Gamma_{A}\right\}\right)$ is called $H$ convex if for each $A \in \mathcal{F}(D), \Gamma_{A} \subset D$.

We shall introduce a condition of generalised Zima type in the following way.

DEFINITION 3. Let $\left(X,\left\{\Gamma_{A}\right\}\right)$ be an $B$-space with a uniformity $\mathcal{V}$ and let $K$ be a nonempty subset of $X$. We say that $K$ is of generalised Zima type if for every

Received 27 July 1995

Copyright Clearance Centre, Inc. Serial-fee code: 0004-9729/96 \$A2.00+0.00. 
$V \in \mathcal{V}$ there exists $U \in \mathcal{V}$ such that for every $D \in \mathcal{F}(K)$ and every $H$-convex subset $M$ of $K$ the following implication holds:

$$
M \cap U(z) \neq \emptyset, \text { for every } z \in D \quad \Rightarrow \quad M \cap V(u) \neq \emptyset \text {, for every } u \in \Gamma_{D} .
$$

REMARK. If $K=X$ and $U=V$, for every $V \in \mathcal{V}$, then $X$ is of generalised Zima type if $X$ is a l. c. $H$-space in the sense of [4].

EXAMPLE. Let $X$ be a topological vector space with a fundamental system of neighbourhoods of zero $\mathcal{V}$ and let $K \subset X$ be of Zima type, that is, for every $V \in \mathcal{V}$ there exists $U \in \mathcal{V}$ such that

$$
\operatorname{co}(U \cap(K-K)) \subseteq V
$$

where $c o$ is the convex hull operation.

We shall prove that (2) implies (1).

Let (2) hold and $D \in \mathcal{F}(K)$ and $M \subseteq K$, where $M$ is convex. Suppose that

$$
M \cap(z+U) \neq \emptyset, \text { for every } z \in D .
$$

If $D=\left\{z_{1}, z_{2}, \ldots, z_{n}\right\}$, it follows from (3) that there exists $\left\{v_{1}, v_{2}, \ldots, v_{n}\right\} \subseteq M$ such that

$$
v_{i} \in M \cap\left(z_{i}+U\right), i \in\{1,2, \ldots, n\} .
$$

Hence, there exists $\left\{w_{1}, w_{2}, \ldots, w_{n}\right\} \subseteq U$ such that $v_{i}=z_{i}+w_{i}, i \in\{1,2, \ldots, n\}$. If $u \in \Gamma_{D}=\operatorname{coD}$ then

$$
u=\sum_{i=1}^{n} \lambda_{i} z_{i}, \lambda_{i} \geqslant 0, i \in\{1,2, \ldots, n\}, \sum_{i=1}^{n} \lambda_{i}=1,
$$

and so:

$$
\sum_{i=1}^{n} \lambda_{i} v_{i}=\sum_{i=1}^{n} \lambda_{i} z_{i}+\sum_{i=1}^{n} \lambda_{i} w_{i} \in u+c o(U \cap(K-K)) \subseteq(u+V) \cap M .
$$

From this it follows that $M \cap(u+V) \neq \emptyset$, for every $u \in \operatorname{co} D$.

Now, we shall give an example of a subset of Zima type in a non locally convex topological vector space.

Let $S(0,1)$ be the space of all the equivalence classes of real measurable functions on $[0,1]$, and for every $\tilde{x} \in S(0,1)$ let

$$
\|\tilde{x}\|=\int_{0}^{1} \frac{|x(t)|}{1+|x(t)|} \mu(d t),\{x(t)\} \in \tilde{x} .
$$


Then $\|\cdot\|$ is a paranorm $(\|\cdot\|$ is not homogeneous) and $S(0,1)$ is a non locally convex topological vector space in which the fundamental system of neighbourhoods of zero is given by the family $\mathcal{V}=\left\{V_{e}\right\}_{e>0}$ where

$$
V_{\varepsilon}=\{x ;\|x\|<\varepsilon\} .
$$

Convergence in this topology coincides with convergence in measure.

Let $\alpha>0$ and $K_{\alpha} \subset S(0,1)$ be defined by

$$
K_{\alpha}=\{\tilde{x} ; \tilde{x} \in S(0,1) ;|x(t)| \leqslant \alpha, t \in[0,1]\} .
$$

It can be shown that for every $s \in[0,1]$ and every $\tilde{x}, \tilde{y} \in K_{\alpha}$ :

$$
\|s(\tilde{x}-\tilde{y})\| \leqslant(1+2 \alpha) s\|\tilde{x}-\tilde{y}\|=C\left(K_{\alpha}\right) s\|\tilde{x}-\tilde{y}\|,
$$

where $C\left(K_{\alpha}\right)=1+2 \alpha>1$. From (4) it follows that

$$
\operatorname{co}\left(V_{\varepsilon /\left(C\left(K_{\alpha}\right)\right)} \cap\left(K_{\alpha}-K_{\alpha}\right)\right) \subset V_{\varepsilon}, \text { for every } \varepsilon>0
$$

which means that $K_{\alpha}$ is of Zima type, for every $\alpha>0$.

\section{AN ALMOST FiXed POINT THEOREM}

If $\left(X ;\left\{\Gamma_{A}\right\}\right)$ is an $H$-space with uniformity $\mathcal{V}, K \subset X$ and $F: K \rightarrow 2^{X}$ then $F$ has a $V$-almost point $(V \in \mathcal{V})$ if there exists $y \in K$ such that

$$
F(y) \cap V(y) \neq \emptyset .
$$

THEOREM 1. Let $\left(X ;\left\{\Gamma_{A}\right\}\right)$ be an $H$-space with uniformity $\mathcal{V}$ and let $K$ be a nonempty, convex and precompact subset of $X$. Let $F: K \rightarrow 2^{X}$ be a lower semicontinuous mapping such that $F(x) \cap K \neq \emptyset$ for every $x \in K$, and $F(x)$ is $H$-convex for every $x \in K$. If $K \cup F(K)$ is of generalised Zima type then $F$ has a $V$-almost fixed point, for every $V \in \mathcal{V}$.

Proof: Let $V \in \mathcal{V}$ and $U \in \mathcal{V}$ be such that (1) holds for every $D \in \mathcal{F}(K \cup F(K))$ and every $H$-convex subset $M \subset K \cup F(K)$. We shall suppose that every $V \in \mathcal{V}$ is open. For every $x \in K$ let

$$
G(x)=\{y ; y \in K, F(y) \cap U(x)=\emptyset\} .
$$

Since $F$ is lower semicontinuous and $U(x)$ is open it follows that $G(x)$ is closed for every $x \in K$. From the precompactness of $K$ it follows that there exists $D_{1} \in \mathcal{F}(K)$ such that

$$
K \subseteq \bigcup_{z \in D_{1}} U(z)
$$


Since for every $x \in K, F(x) \cap K \neq \emptyset$, it follows from (5) that $F(x) \cap\left(\bigcup_{z \in D_{1}} U(z)\right) \neq \emptyset$. Hence $\bigcap_{z \in D_{1}} G(z)=0$. From this we conclude that there exists $D_{2} \subset D_{1}$ such that

$$
\Gamma_{D_{2}} \notin \bigcup_{z \in D_{2}} G(z)
$$

This means that there exists $y \in \Gamma_{D_{2}}$ such that $y \notin G(z)$, for every $z \in D_{2}$, this means that

$$
F(y) \cap U(z) \neq \emptyset, \text { for every } z \in D_{2} .
$$

Since $F(y) \subseteq K \cup F(K)$ and $K \cup F(K)$ is of generalised Zima type, it follows that $F(y) \cap V(y) \neq \emptyset$, since $y \in \Gamma_{D_{2}}$. Hence $y$ is a $V$-almost fixed point for $F$.

\section{A BEST APPROXIMATION THEOREM}

In this part of the paper we suppose that $\left(X ;\left\{\Gamma_{A}\right\}\right)$ is a metrisable $H$-space with metric $d$.

If $\emptyset \neq K \subset X$ and $F: K \rightarrow 2^{X}$ we say that $F$ satisfies the $Z_{\delta}$-condition on $K$ if for every $y \in K$ and every $\varepsilon>0$ :

$$
\operatorname{co}(L(F(y), \delta(\varepsilon)) \cap K) \subset L(F(y), \varepsilon)
$$

where $\delta:[0, \infty) \rightarrow[0, \infty)$ and for $M \subset X, r>0$

$$
L(M, r)=\{x ; x \in X, d(x, M)<r\} .
$$

EXAMPLE 2. Let $(X,\|\|)$ be a paranormed space, $\emptyset \neq K \subset X, F: K \rightarrow 2_{c o}^{X}$ and suppose there exists $C>0$ such that for every $s \in[0,1]$

$$
\|s(u-v)\| \leqslant C s\|u-v\|, \text { for every } u \in K, v \in F(K) .
$$

We shall prove that (7) implies (6) for $\delta(\varepsilon)=\varepsilon / C$. Let $z \in \operatorname{co}(L(F(y), \delta(\varepsilon)) \cap K)$. This means that there exists $\left\{x_{1}, x_{2}, \ldots, x_{n}\right\} \in L(F(y), \delta(\varepsilon)) \cap K$ such that $z=\sum_{i=1}^{n} \lambda_{i} x_{i}$, $\lambda_{i} \geqslant 0, i \in\{1,2, \ldots, n\}, \sum_{i=1}^{n} \lambda_{i}=1$.

Hence $x_{i} \in K$ and $d\left(x_{i}, F(y)\right)<\delta(\varepsilon)$, for every $i \in\{1,2, \ldots, n\}$. Hence, there exists $v_{i} \in F(y), i \in\{1,2, \ldots, n\}$ such that $\left\|x_{i}-v_{i}\right\|<\delta(\varepsilon)$, for every $i \in\{1,2, \ldots, n\}$.

Then (7) implies

$$
\left\|\sum_{i=1}^{n} \lambda_{i} x_{i}-\sum_{i=1}^{n} \lambda_{i} v_{i}\right\| \leqslant C \sum_{i=1}^{n} \lambda_{i}\left\|x_{i}-v_{i}\right\|<C \cdot \frac{\varepsilon}{C}=\varepsilon .
$$

Since $F(y)$ is convex, $\sum_{i=1}^{n} \lambda_{i} v_{i} \in F(y)$ and so $d(z, F(y)) \leqslant\left\|y-\sum_{i=1}^{n} \lambda_{i} v_{i}\right\|<\varepsilon$, which means that $z \in L(F(y), \varepsilon)$. 
Example 3. If $(X, d, W)$ is a Takahashi convex metric space (as defined by Takahashi in [15]) with continuous $W$ and

$$
d\left(W\left(x_{1}, x_{2}, \lambda\right), W\left(z_{1}, z_{2}, \lambda\right)\right) \leqslant \lambda d\left(x_{1}, z_{1}\right)+(1-\lambda) d\left(x_{2}, z_{2}\right)
$$

then for any $\left\{x_{1}, x_{2}, \ldots, x_{n}\right\} \subset X$, any $y \in \operatorname{cow}_{W}\left\{x_{1}, x_{2}, \ldots, x_{n}\right\}$ and any $W$-convex set $A$ :

$$
d(y, A) \leqslant \min _{1 \leqslant i \leqslant n} d\left(x_{i}, v_{i}\right)
$$

for arbitrary $v_{i} \in A(i \in\{1,2, \ldots, n\})$, see $[11]$.

In this case (6) holds for $\delta(\varepsilon)=\varepsilon$, for every $\varepsilon>0$. Indeed if $M$ is an arbitrary convex set in $X$ then $L(M, \varepsilon)$ is a convex set as well. Suppose that $x_{i} \in L(M, \varepsilon)$, $i \in\{1,2, \ldots, n\}$. Then $d\left(x_{i}, M\right)<\varepsilon$, for every $i \in\{1,2, \ldots, n\}$ and so there exists $v_{i} \in M, i \in\{1,2, \ldots, n\}$ such that $d\left(x_{i}, v_{i}\right)<\varepsilon, i \in\{1,2, \ldots, n\}$. From (8) it follows that for every $y \in \operatorname{cow}_{W}\left\{x_{1}, x_{2}, \ldots, x_{n}\right\}$ :

$$
d(y, M)<\varepsilon
$$

and so $y \in L(M, \varepsilon)$, which means that $L(M, \varepsilon)$ is a $W$-convex set.

In the proof of the next theorem we shall use the following result of Horvath [12]:

Let $(M, d)$ be a complete metric space and let $\left\{F_{i}\right\}_{i \in I}$ be a family of closed subsets in $M$. If the family $\left\{F_{i}\right\}_{i \in I}$ has the finite intersection property and $\underset{i \in I}{\inf } \alpha\left(F_{i}\right)=0$, where $\alpha$ is the Kuratowski measure of noncompactness, then $\bigcap_{i \in I} F_{i}$ is compact and nonempty.

TheOrem 2. Let $\left(X ;\left\{\Gamma_{A}\right\}\right)$ be a metrisable $H$-space with metric $d, \emptyset \neq M$ an $H$-convex and complete subset of $X$, and let $F: M \rightarrow \mathcal{K}(X)$ (the family of all nonempty $H$-convex and compact subsets of $X$ ) be a continuous mapping which satisfies the $Z_{\delta}$ condition on $M$, where $\delta$ is continuous and

$$
\inf _{x \in M} \alpha[\{y ; y \in M, \delta(d(y, F(y))) \leqslant d(x, F(y))\}]=0 .
$$

Then there exists $y_{0} \in M$ such that

$$
\delta\left(d\left(y_{0}, F\left(y_{0}\right)\right)\right) \leqslant \inf _{z \in M} d\left(x, F\left(y_{0}\right)\right) .
$$

Proof: Let $G(x), x \in M$, be defined in the following way:

$$
G(x)=\{y ; y \in M, \delta(d(y, F(y))) \leqslant d(x, F(y))\} .
$$


We shall prove that $G$ is an $H$-KKM mapping, that is, that for every $D=\left\{x_{1}, x_{2}, \ldots, x_{n}\right\} \subseteq$ $M$

$$
\Gamma_{D} \subseteq \bigcup_{z \in D} G(z)
$$

Suppose that $\Gamma_{D} \notin \bigcup_{z \in D} G(z)$. Then there exists $y \in \Gamma_{D}$ such that $y \notin G\left(x_{i}\right)$ for every $i \in\{1,2, \ldots, n\}$. This means that

$$
\delta(d(y, F(y)))>d\left(x_{i}, F(y)\right), \text { for every } i \in\{1,2, \ldots, n\},
$$

and so $x_{i} \in L(F(y), \delta(d(y, F(y)))) \cap M$, for every $i \in\{1,2, \ldots, n\}$. This implies that

$$
\begin{gathered}
y \in \operatorname{co}(L(F(y), \delta(d(y, F(y)))) \cap M) \\
\subset L(F(y), d(y, F(y)))
\end{gathered}
$$

which means that $d(y, F(y))<d(y, F(y))$. This is a contradiction. Hence (10) holds and $G$ is an $H$-KKM mapping.

In order to prove that $G(x)$ is closed for every $x \in M$ we shall prove that the mapping $y \mapsto \delta(d(y), F(y))(y \in M)$ is lower semicontinuous and for every $x \in M$, the mapping $y \mapsto d(x, F(y))$ is upper semicontinuous. Let $\gamma>0$ and

$$
\begin{aligned}
& P_{\boldsymbol{\gamma}}=\{y ; y \in M, \delta(d(y, F(y)))>\gamma\} \\
& Q_{\gamma}=\{y ; y \in M, d(x, F(y))<\gamma\} .
\end{aligned}
$$

We prove that $P_{\boldsymbol{\gamma}}$ and $Q_{\boldsymbol{\gamma}}$ are open.

Since $y \mapsto(y, F(y))$ is upper semicontinuous and

$$
P_{\gamma}=\{y ; y \in M,(y, F(y)) \subseteq\{(z, v) ;(z, v) \in M \times X ; \delta(d(z, v))>\gamma\}\}
$$

it follows that $P_{\gamma}$ is open.

Analogously, since $F$ is lower semicontinuous and

$$
Q_{\gamma}=\{y ; y \in M, F(y) \cap\{v ; v \in X, d(x, v)<\gamma\} \neq \emptyset\}
$$

therefore $Q_{\gamma}$ is open.

Hence $G(x)$ is closed, for every $x \in M$, and since $G$ is an $H$-KKM mapping it follows that $\{G(x)\}_{x \in M}$ has the finite intersection property. From (9) it follows that $\bigcap_{x \in M} G(x) \neq \emptyset$. If $y_{0} \in \bigcap_{x \in M} G(x)$ then

$$
\delta\left(d\left(y_{0}, F\left(y_{0}\right)\right)\right) \leqslant \inf _{x \in M} d\left(x, F\left(y_{0}\right)\right) .
$$


Corollary 1 . Let $(X, d, W)$ be a Takahashi convex metric space with continuous $W$ such that (a) holds. Let $\emptyset \neq M$ be a convex and complete subset of $X$ and let $F: M \rightarrow \mathcal{K}(X)$ be a continuous mapping such that

$$
\inf _{x \in M} \alpha[\{y ; y \in M, d(y, F(y)) \leqslant d(x, F(y))\}]=0
$$

Then there exists $y_{0} \in M$ such that

$$
d\left(y_{0}, F\left(y_{0}\right)\right)=\inf _{x \in M} d\left(x, F\left(y_{0}\right)\right)
$$

\section{REFERENCES}

[1] C. Bardaro and R. Ceppitelli, 'Some further generalizations of Knaster-KuratowskiMazurkiewicz theorem and minimax inequalities', J. Math. Anal. Appl. 132 (1988), 484-490.

[2] S.S. Chang and L. Yang, 'Section theorems on $H$-spaces with applications', J. Math. Anal. Appl. 179 (1993), 214-231.

[3] X.P. Ding, 'An existence theorem for maximizable $H$-quasiconcave functions', Acta Math. Sinica 36 (1993), 273-279.

[4] X.P. Ding, 'Equilibrium existence theorems of abstract economics in $H$-spaces', Indian $J$. Pure Appl. Math. 25 (1994), 303-317.

[5] X.P. Ding and K-K. Tan, 'Generalizations of KKM theorem and applications to best approximations and fixed point theorems', Southeast Asian Bull. Math. 17 (1993), 139-150.

[6] X.P. Ding, W.K. Kim and K-K. Tan, 'A new minimax inequality on $H$-spaces with applications', Bull. Austral. Math. Soc. 41 (1990), 457-473.

[7] X.P. Ding, W.K. Kim and K-K. Tan, 'Applications of a minimax inequality on $H$-spaces', Bull. Austral. Math. Soc. 41 (1990), 475-485.

[8] O. Hadžić, 'Some fixed point and almost fixed point theorems for multivalued mappings in topological vector spaces', Nonlinear Anal. Theory, Methods, Appl. 5 (1981), 1009-1019.

[9] O. Hadžić, 'Fixed point theorems in not necessarily locally convex spaces', in Lecture Notes in Mathematics 943 (Springer Verlag, Berlin, Heidelberg, New York, 1982), pp. 118-130.

[10] O. Hadžić, 'Some properties of measures of noncompactness in paranormed spaces', Proc. Amer. Math. Soc. 102 (1988), 843-849.

[11] O. Hadžić, 'On best approximations for multivalued mappings in pseudoconvex metric spaces', Univ.u Novom Sadu Zb. Rad. Prirod.-Mat. Fak. Ser. Mat. 24 (1994), 1-12.

[12] C. Horvath, 'Measure of non-compactness and multivalued mappings in complete metric topological vector spaces', J. Math. Anal. Appl. 180 (1985), 403-408.

[13] H.M. Li and X.P. Ding, 'Versions of the KKM theorem in $H$-spaces and their applications', Sichuan Shifan Dexue Xuebao Ziran Kexue Ban 16 (1993), 21-27. 
[14] S. Park, 'The Brouwer and Schauder fixed point theorems for spaces having certain contractible subsets', Bull. Korean Math. Soc. 30 (1993), 83-89.

[15] W.A. Takahashi, 'A convexity in metric spaces and nonexpansive mappings', I. Kodai Math. Sem. Rep. 29 (1977), 62-70.

[16] A. Tarafdar, 'A fixed point theorem in $H$-spaces and related results', Bull. Austral. Math. Soc 42 (1990), 133-140.

[17] S.S. Zhang, S.K. Kang and L. Yang, 'Coincidence point theorems for set-valued mappings in $H$-spaces and minimax inequalities', J. Chengdu Univ. Sci. Tech. 3 (1993), 57-61.

\author{
University of Novi Sad \\ Department of Mathematics \\ 21000 Novi Sad \\ Trg Dositeja Obradovica 4 \\ Yugoslavia
}

